Published in final edited form as:

Anal Chem. 2016 November 15; 88(22): 11139-11146. doi:10.1021/acs.analchem.6b03234.

\title{
Heavy Sugar and Heavy Water Create Tunable Intact Protein Mass Increases for Quantitative MS in any Feed and Organism
}

\author{
Jeniffer V. Quijada ${ }^{\dagger}, \|$, Nicholas D. Schmitt ${ }^{\dagger}, \|$, Joseph P. Salisbury ${ }^{\dagger, \|}$, Jared R. Auclair ${ }^{\dagger, \|}$, and \\ Jeffrey N. Agar ${ }^{\star}, \dagger, \|, \S$ \\ tDepartment of Chemistry and Chemical Biology, Northeastern University, 360 Huntington \\ Avenue, Boston, MA, 02115 \\ "Barnett Institute of Chemical and Biological Analysis, Northeastern University, 360 Huntington \\ Avenue, Boston, MA, 02115 \\ $\S$ Department of Pharmaceutical Sciences, Northeastern University, 360 Huntington Avenue, \\ Boston, MA, 02115
}

\begin{abstract}
Stable isotope labeling techniques for quantitative top-down proteomics face unique challenges. These include unpredictable mass shifts following isotope labeling, which impedes analysis of unknown proteins and complex mixtures; and exponentially greater susceptibility to incomplete isotope incorporation, manifesting as broadening of labeled intact protein peaks. Like popular bottom-up isotope labeling techniques, most top-down labeling methods are restricted to defined media/feed as well as amino acid auxotrophic organisms. We present a labeling method optimized for top-down proteomics that overcomes these challenges. We demonstrated this method through the spiking of ${ }^{13} \mathrm{C}$-sugar or ${ }^{2} \mathrm{H}$-water into standard laboratory feedstocks, resulting in Tunable Intact Protein Mass Increases (TIPMI). After mixing of labeled and unlabeled samples, direct comparison of light and heavy peaks allowed for the relative quantitation of intact proteins in three popular model organisms, including prokaryotic and eukaryotic microorganisms, and an animal. This internal standard method proved to be more accurate than label-free quantitation in our hands. Advantages over top-down SILAC include working equally well in nutrient-rich media, conceivably expanding applicability to any organism and all classes of biomolecules; not requiring high resolving power MS for quantitation; and being relatively inexpensive.
\end{abstract}

\footnotetext{
*Corresponding author, j.agar@ northeastern.edu, phone: 617.373.5909.

Author Contributions

J.N.A. and J.R.A. designed the research. J.V.Q. performed the experiments. J.V.Q., N.D.S., and J.P.S. performed the calculations and data analysis. J.N.A., N.D.S. and J.V.Q. wrote the manuscript; all authors contributed substantial input and editing.

Notes

The authors declare no competing financial interest.

Supporting Information

The Supporting Information is available free of charge on the ACS Publications website.

Supporting Methods, Supporting Figures and Tables (PDF)
} 


\section{Introduction}

Advances in proteomics methods enable quantitative biological studies to be more accessible, accurate, and robust. While the most accurate methods have historically been those using stable isotope labeling (SIL), the most accessible methods have been label-free approaches. This is due to the high cost of labeling reagents and the limited number of organisms applicable to SIL methods. In this study, we aimed to bridge this gap by introducing an inexpensive SIL method that allows for comparative top-down proteomics.

Techniques for quantitative proteomics include label-free and isotope dilution approaches employing chemical or metabolic labeling. ${ }^{1}$ Label-free and chemical techniques are amenable to any preparation, but do not correct for all variation in sample preparation. In addition, label-free techniques cannot be multiplexed and do not correct for variations in Liquid Chromatography Mass Spectrometry (LC-MS) analysis. Metabolic labeling creates heavy proteins and peptides that serve as internal standards, accounting for variation in sample preparation and LC-MS, and has been used extensively for expression proteomics and protein anabolism studies. Each previous metabolic labeling strategy addresses only a subset of organisms. Incorporation of ${ }^{13} \mathrm{C}$ using labeled ${ }^{13} \mathrm{CO}_{2}$, and ${ }^{15} \mathrm{~N}$ using labeled ${ }^{15} \mathrm{NH}_{4}{ }^{+}$(atom-replacement) is successful for plants and some microorganisms, whereas stable isotope labeling by amino acids in cell culture (SILAC) ${ }^{2}$ is applicable to cells that can be grown in defined media/feed, and that are auxotrophic for the labeled amino acid. Controlled feedstocks have enabled stable isotope labeling of mammals (SILAM) ${ }^{3}$ and SILAC animals have been created. ${ }^{4}$ However, such nominally incompatible organisms receive a feed of labeled microorganisms, ${ }^{5}$ requiring non-standard, less nutritious, and sometimes toxic diets. 6

The first metabolic labeling proteomics studies of any kind compared intact proteins from microbial cells grown on ${ }^{13} \mathrm{C}$ - and ${ }^{15} \mathrm{~N}$-depleted media, to those grown on natural abundance media. ${ }^{7}$ A comparative top-down study in yeast employed complete replacement of ${ }^{14} \mathrm{~N}$ with ${ }^{15} \mathrm{~N}$ using $\left({ }^{15} \mathrm{NH}_{4}{ }^{+}\right)_{2} \mathrm{SO}_{4} \cdot{ }^{8}$ In addition, the first application of SILAC involved counting the number of leucines in intact proteins to provide more accurate protein identification (but not to quantify). ${ }^{9}$ This approach was subsequently extended to quantitation of endoproteinasedigested proteins ${ }^{10}$ and became one of the most popular methods for quantitative proteomics (SILAC). Although it has been shown that SILAC is compatible with top-down proteomics in a few proof-of-concept studies, ${ }^{11,12}$ it results in peak broadening and peak asymmetry. Due to mass shifts that vary widely as a function of arginine and lysine content, intact protein SILAC should also result in difficulties in assigning light-heavy peak pairs in complex samples of unknown proteins. To our knowledge, SILAC has not been used to discover differences in expression in a quantitative top-down proteomics experiment. With sufficient expertise and equipment, label-free top-down quantitation is effective. ${ }^{13,14}$ However, as will be demonstrated here, for high variability intact protein LC-MS/MS analysis, the use of internal standards produced by metabolic labeling can improve accuracy and precision.

As opposed to the complete isotope replacement techniques outlined above, replacing a certain percentage of isotopes is less expensive and affords the labeling of additional 
organisms. Mass isotopomer distribution analysis (MIDA) focuses on the rates of synthesis of biological polymers ${ }^{15}$, including proteins. ${ }^{16}$ Such partial incorporation of ${ }^{13} \mathrm{C}$ through labeled sugar has been demonstrated for peptide quantitation for use in characterizing protein expression dynamics in vitro. ${ }^{17}$ Partial incorporation of deuterated water has been used for studying proteome dynamic turnover in vivo. ${ }^{18,19}$ Provided that the identity of the peptides are known, subtle modification of isotope ratio proteomics (SMIRP), ${ }^{20}$ using ${ }^{13} \mathrm{C}$ bicarbonate (incorporated as $\mathrm{CO}_{2}$ ), and related techniques can measure differences in protein expression. ${ }^{21}$ Stable-isotope probing (SIP) studies feed isotopically labeled metabolic precursors to examine metabolic competence and flux. SIP has been used successfully for protein labeling. ${ }^{22}$ However, to our knowledge, partial metabolic labeling techniques have not been employed in comparative top-down studies.

Microbes are problematic for SILAC due to their ability, as prototrophs, to synthesize amino acids that compete for incorporation with the labeled amino acid. Animals are problematic for SILAC because, as auxotrophs for essential amino acids, ideal growth requires nutrientrich media that contains unlabeled amino acids that compete with labeled amino acids. ${ }^{13} \mathrm{C}$ from glucose and ${ }^{2} \mathrm{H}$ from deuterium oxide are metabolized through glycolysis and hydrolysis, respectively, which are shared by all life. ${ }^{23}$ Using three model organisms yeast, bacteria, and fruit flies we have shown that a varying amount of labeled sugar and water can be spiked into a range of feedstocks and incorporated into proteins, creating tunable intact protein mass increases (TIMPI). This partial metabolic labeling method employs atomreplacement with heavy isotopes to increase the mass of biomolecules until their MS peaks can be distinguished from natural abundance peaks. These heavy intact protein peaks can then be used for isotope dilution mass spectrometry upon mixing with natural abundance samples. This study represents our initial efforts to blend top-down proteomics and previous labeling approaches into a new, simple, and inexpensive way to measure differences in protein expression.

\section{Materials and Methods \\ Saccharomyces cerevisiae (yeast) growth}

Yeast strain S288C (a wild-type strain) of Saccharomyces cerevisiae was acquired from ATCC. Standard methods for the growth of yeast were applied and are described elsewhere. ${ }^{24}$ Briefly, natural YPD was prepared by adding $1 \mathrm{~g}$ yeast extract (BD Bacto Yeast Extract Ref. 212750), $2 \mathrm{~g}$ tryptone (BD Bacto tryptone Ref.211705), and $2 \mathrm{~g}$ dextrose (D-Glucose, natural abundance from Fisher D16-500) to $100 \mathrm{~mL}$ of ultrapure water followed by autoclaving. In order to incorporate ${ }^{13} \mathrm{C}$, YPD was prepared as above, replacing the dextrose (20 mg/1mL) with ${ }^{13} \mathrm{C}_{6}$-dextrose (D-Glucose Cambridge Isotope Laboratory U-13C6, $99 \%$ ref. CLM-1396-1) and prepared in $3.5 \mathrm{~mL}$ batches. Day 1: S288C yeast cells were grown on YPD agar plates, a single colony was used to inoculate a $5 \mathrm{~mL}$ culture of YPD (natural abundance), and was grown overnight at $30{ }^{\circ} \mathrm{C}$ aerobically using a shaker incubator (New Brunswick Scientific C24) at 220 RPM. Day 2: the yeast solution from day 1 was diluted in natural abundance YPD until an $\mathrm{OD}_{600}$ of $\sim 0.6$ and was then used to inoculate $(1: 100)$ natural abundance YPD or ${ }^{13} \mathrm{C}_{6}$-dextrose YPD growth media. These dilutions were split into 
$1 \mathrm{~mL}$ aliquots of labeled or natural abundance YPD and cultured for 30 hours (late log phase growth) at $30^{\circ} \mathrm{C}$.

\section{Yeast sample preparation}

All sample handling was performed at $4{ }^{\circ} \mathrm{C}$ using an ice bath or fridge unless otherwise stated. Cells from a $1 \mathrm{~mL}$ culture were pelleted at $14,000 \mathrm{rpm}$ for 10 minutes at $4{ }^{\circ} \mathrm{C}$ and washed with $300 \mu \mathrm{L}$ of cold lysis buffer ( $25 \mathrm{mM}$ Tris, $\mathrm{pH} 7.4$, and $0.1 \mathrm{mM}$ EDTA, with one protease inhibitor cocktail table - SIGMAFAST Tablets Ref. S8820). This solution was centrifuged at $14,000 \mathrm{rpm}$ for 10 minutes at $4{ }^{\circ} \mathrm{C}$ and the supernatant was discarded. To evaluate the variability and dynamic range of TIPMI, resuspended yeast (in $100 \mu \mathrm{L}$ lysis buffer) grown in natural abundance media were mixed at a range of volume ratios with yeast grown in labeled media. In total, nine relative concentrations were evaluated, including ratios of 8:1, 4:1, 2:1, 1:1, 1:2, 1:4, and 1:8 (Light:Heavy), as well as two controls (natural abundance-only and labeled-only, Figure S1). The cells were immediately sonicated for 10 seconds followed by a 30-second rest on ice (Sonicator model W-220F). This cycle was performed three times. After cell lysis, the solution was cleared by centrifugation at 14,000 rpm for 10 minutes at $4{ }^{\circ} \mathrm{C}$ and the supernatant was transferred to a clean tube.

\section{Protein precipitation}

Contaminants (detergents, lipids, salts, buffer components) were removed by a protein precipitation method previously described. ${ }^{25}$ Briefly, methanol, chloroform, and water were added at a ratio of 4:1:3 volumes, sequentially, with a 10-second vortex between each solvent addition. The precipitated proteins were washed with three volumes of methanol and in all cases; the liquid was carefully removed using a gel loading pipette tip and vacuum aspiration. For Escherichia coli (E. coli) and Drosophila samples, the precipitated protein was kept in the mix of methanol:chloroform:water at $-20{ }^{\circ} \mathrm{C}$ for $10 \mathrm{~min}$ before centrifuge and wash, which improved the reproducibility of protein precipitation. The supernatant was removed by vacuum aspiration and the precipitate was dried in the hood (10 min) until the remaining solvent evaporated, and stored at $-80^{\circ} \mathrm{C}$ until LC-MS analysis.

\section{LC-MS analysis}

The precipitated yeast proteins were re-suspended in $30 \mu \mathrm{L}$ of $10 \% \mathrm{v} / \mathrm{v}$ acetonitrile with $0.1 \% \mathrm{v} / \mathrm{v}$ formic acid by breaking the precipitate with a spatula for 10 seconds and sonicating in a water bath for 1 minute. The resuspension was centrifuged for 20 minutes at $14,000 \mathrm{rpm}$ at $4{ }^{\circ} \mathrm{C}$, and then $10 \mu \mathrm{L}$ were injected for LC-MS. Drosophila and E. coli precipitated proteins were resuspended at $-20^{\circ} \mathrm{C}$ with $15 \mu \mathrm{L}$ of pre-chilled $80 \% \mathrm{v} / \mathrm{v}$ formic acid. ${ }^{26}$ After 2 minutes, the sample was mixed by pipetting and kept at $-20^{\circ} \mathrm{C}$ for 8 minutes (a total of 10 minutes). $15 \mu \mathrm{L}$ of pre-cooled water $\left(4^{\circ} \mathrm{C}\right)$ was then added, mixed and centrifuged for 20 minutes at $14,000 \mathrm{rpm} 4{ }^{\circ} \mathrm{C}$, and used for LC-MS analysis. LC-MS analysis was performed on a Waters NanoAcquity UPLC by injecting $10 \mu \mathrm{L}$ of protein resuspension onto a pre-filter $0.5 \mu \mathrm{m}$ PEEK (IDEX A-356) and trap column $3 \mathrm{~cm} x 150 \mu \mathrm{m}$ i.d. packed in house with PLRP-S beads (Agilent Part \# PL1512-1502, $1000 \AA$ A pore size, 5 $\mu \mathrm{m}$ particle size). ${ }^{27}$ The mobile phase A was water with $0.1 \% \mathrm{v} / \mathrm{v}$ formic acid (Sigma); mobile phase B was acetonitrile, $0.1 \% \mathrm{v} / \mathrm{v}$ formic acid (Sigma). The sample was loaded at $10 \% \mathrm{v} / \mathrm{v}$ B for 15 minutes with a flow rate of $2 \mu \mathrm{L} / \mathrm{min}$ in the trap column and transferred to 
an inline PLRP-S analytical column $6 \mathrm{~cm} \times 150 \mu \mathrm{m}$ i.d. packed in house, and proteins were eluted using a gradient from 10-32\% v/v B in 46 minutes. The column was equilibrated with $10 \% \mathrm{v} / \mathrm{v}$ B for 21 minutes prior to the gradient running and washed with $100 \% \mathrm{v} / \mathrm{v} \mathrm{B}$ for 10 minutes after the gradient. Quantitative LC-MS studies utilized an Impact HD Q-TOF (Bruker Daltonics). Although these studies were performed using a Q-TOF mass spectrometer, higher resolution Fourier transform mass spectrometry may be necessary for more complex mixtures. Base peak separation was achieved following chloroform/methanol protein precipitation with a mixture of labeled and unlabeled proteoforms co-eluting (Figure 1). Samples were analyzed using Q-TOF source parameters. 5.0 L/min drying gas flow $\left(200{ }^{\circ} \mathrm{C}\right.$ ) with the capillary voltage set to $4.0 \mathrm{kV}$. The end plate offset was at $500 \mathrm{~V}$ and nebulizer at $14.5 \mathrm{psi}$. The MS mass range was $\mathrm{m} / \mathrm{z} 500$ to 3000 and spectra were acquired by averaging two scans at an acquisition rate of $1 \mathrm{~Hz}$.

\section{Data Analysis for Relative Quantitation}

\section{Peak nomenclature}

Our experimental workflow involved mixing live natural abundance yeast with labeled yeast, which could (and did) result in slight labeling of the natural abundance yeast between the times the natural abundance and labeled samples were mixed and lysed. To avoid any ambiguity "natural abundance" refers to a peak observed in the natural abundance control, whereas "light" refers to the corresponding peak in a mixture. By analogy, "labeled" refers to a peak observed in the labeled control, whereas "heavy" refers to the corresponding peak in a mixture.

\section{Quantitation}

Using LabVIEW, we were able to upload the low resolving power LC-MS deconvoluted data from the mixed sample and two controls, one natural and one labeled. Light-Heavy peak pairs were recognizable by manual inspection, and the mass range was defined for each peak pair manually. These mass ranges were used to extract mass chromatograms as a function of time and determine the LC elution time width for mixed samples and controls (which ranged from 0.75 to 3 minutes). The selection of mass range and elution time are manually adjusted for each peak pair. The isotopomer distributions were validated as a light-heavy peak pair if they co-eluted in mixed sample, had the expected differences in mass defects (not applicable to larger proteins), and were separated in mass by the correct amount (e.g., the light and heavy peak in this study were separated by the predicted amount) (Figure 1 and Figure S2). Once the peak pair is isolated by mass and time, mass spectra for an L-H pair were then averaged across their elution profile to yield an average spectrum. The average spectrum from given mixture (e.g. $2: 1$ natural abundance: $8.1 \%{ }^{13} \mathrm{C}$ incorporated in yeast) was displayed together with a spectrum of the natural abundance control and labeled "heavy" control for reference. The mass range for the light peak began at the baseline of light peak on the low mass side, to the inflection point between light and heavy peaks. The mass range for the heavy peak began at this inflection until it reached baseline (Figure S3). Peak areas under the curve for light and heavy peaks were used to calculate light/heavy ratios. Subtraction of a natural abundance spectrum (optional, but used here, see below) improved 
accuracy by eliminating the contribution of the area of light post-translational modifications (PTMs) to heavy peaks.

\section{Label-Free Quantitation Details}

For comparison, label-free data analysis was conducted with the same data (light peak areas) used for the TIPMI analysis. A number of literature methods for normalization were attempted. ${ }^{13}$ Normalization to the 21 most-abundant proteins was found to have a lower standard error and higher $\mathrm{R}^{2}$ than both the total ion chromatogram (TIC) and base peak chromatogram (BPC) normalization methods. An analysis of the major sources of error showed that label-free analysis was substantially improved by removing two outlier proteins (notably, one early and one late LC-eluter with highly variable chromatographic retentions). This sans outlier label-free analysis is also shown (Figure S4).

\section{Supporting Methods}

Details of fruit fly and E. coli sample preparation, along with LC-MS/MS, analysis preprocessing, peak picking, accounting for PTMs, and simulations such as those found in Figure 2 are available in the Supporting Information.

\section{Results and Discussion}

\section{Labeled glucose and water can be used to create heavy proteins for isotope dilution MS}

Metabolic incorporation of ${ }^{13} \mathrm{C}$ from glucose into proteins occurs by well-characterized metabolic pathways (Figure S5) and can increase protein mass, as demonstrated in Figure 1. Substituting glucose in YPD with labeled ${ }^{13} \mathrm{C}$ glucose ( $2 \% \mathrm{w} / \mathrm{v}$, a nutrient-rich media) changed the ${ }^{13} \mathrm{C}$ abundance of proteins from $\sim 1.1 \%{ }^{13} \mathrm{C}$ (at natural abundance) to $\sim 9.2 \%{ }^{13} \mathrm{C}$ total incorporation, increasing the masses by $\sim 0.4 \%$ and creating resolved isotopic (isotopologue) distributions for the intact proteins. Based upon our estimate of a $0.36\left[{ }^{13} \mathrm{C} /\right.$ $\left.\left({ }^{12} \mathrm{C}+{ }^{13} \mathrm{C}\right)\right]$ ratio in the growth media, $22 \%$ of the ${ }^{13} \mathrm{C}$ added as sugar was incorporated in proteins. This is expected under (exponential) fermentative growth on glucose, because yeast also convert glucose to energy, expelling ${ }^{13} \mathrm{CO}_{2}$, and ${ }^{13} \mathrm{C}_{2} \mathrm{H}_{6} \mathrm{O}$ (ethanol). In theory, a range of mass shifts (and therefore multiplexing) are available by changing the concentration of ${ }^{13} \mathrm{C}$ glucose (Figure 2), or by decreasing the amount of natural abundance carbon in the media (Figure S6). TIPMI could also be performed in minimal media, offering an expanded range of mass shifts. For example, previous experiments in E. coli. grown on doublydepleted ${ }^{13} \mathrm{C}^{15} \mathrm{~N}$ media ${ }^{28}$ and our experiments in yeast ${ }^{22}$ indicate that ${ }^{13} \mathrm{C}$ total abundance from $0.005 \%$ to $99.5 \%$ are accessible by changing the ${ }^{12} \mathrm{C} /{ }^{13} \mathrm{C}$ glucose ratio in minimal media with glucose as the only carbon source (Figure 2). The successful application of TIPMI using ${ }^{2}$ D-labeled water, in both Drosophila and E. coli are also demonstrated below.

\section{TIPMI complements previous partial labeling techniques}

The fundamental difference between this and previous studies is that labeling regimes that result in overlap between labeled and unlabeled peptides, do not result in overlapping isotope distributions for intact proteins. This is an important distinction because accurate deconvolution of overlapping labeled and unlabeled isotope distributions requires knowledge of the molecule's identity as well as the precise amount of label incorporation. Partial 
labeling with $\mathrm{CO}_{2}$ was demonstrated previously for endoproteinase-derived peptides, but resulted in overlapping isotopic distributions of light and heavy peptide peaks. ${ }^{29}$ This method was then extended to additional labels of heavy nitrogen, using amino acids or ammonium ions, or alternatively deuterium labeling via water. Automated computational workflows were developed to deconvolute the overlapping distributions, and partial metabolic labeling performed comparably to established (full) metabolic labeling methods. ${ }^{30}$ Those interested in applying TIPMI to endoproteinase digests could utilize these computational workflows with the caveat that, unlike TIPMI, previous partial metabolic labeling studies ${ }^{30,31,32}$ require knowledge of the identity of the peptide and the percent of label that was incorporated. ${ }^{30}$ As far as we are aware, this is the first partial metabolic labeling method to use glucose for labeling and analyzing intact proteins, making it broadly applicable, as well as the first to create resolved (nominal mass) isotope distributions for use in quantitation.

\section{TIPMI results in predictable mass shifts}

Assigning peak pairs (the corresponding labeled and unlabeled peaks of a given molecule) is the sine qua non of metabolic labeling MS. While this is not an issue with purified proteins, labeled peaks can be difficult to assign to their unlabeled counterpart in complex spectra (e.g., from LC-MS analyses of complex mixtures). The identification of SILAC peak pairs is compounded by the highly variable, lysine and arginine-dependent, mass shifts of the labeled peaks. TIPMI peak pairs, on the other hand, are more predictably spaced because the $\%{ }^{13} \mathrm{C}\left(\right.$ or ${ }^{2} \mathrm{H}$ ) incorporation in a protein varies less than the $\%$ lysine or $\%$ arginine. Figure 2 illustrates this capability for three co-eluting proteins in the $8 \mathrm{kDa}$ range. Whereas TIPMI heavy labeled peak shifts vary in the range of 25-36 Da, R-SILAC values range from $0 \mathrm{Da}$ (no arginine in the protein) to $24 \mathrm{Da}$. Figure 3 illustrates the predictability of TIPMI peak shifts throughout a broad protein mass range. For example, at $\sim 15 \mathrm{kDa}$, theoretical mass shifts for TIPMI-labeled-yeast vary by only $0.05 \%$ (range of $56-63 \mathrm{Da}$ ), whereas theoretical K-SILAC peak shifts vary by $0.47 \%$ Daltons (range of $35-105 \mathrm{Da}$ ). A similar observation was made from examination of R-SILAC data (Figure S7). Therefore, in theory, TIPMI peak pair distances of intact proteins vary by $1 / 10^{\text {th }}$ the value of SILAC peak pair. This advantage only applies to intact proteins, of course, because digestion with trypsin results in only one label (lysine or arginine) per peptide.

In the event that some amino acids are labeled at different rates than others, ${ }^{33}$ and equilibrium labeling is not achieved, a bias similar to that observed with SILAC could be introduced to TIPMI. To address this potential caveat, the experimentally observed range of TIPMI peak shifts were compared to theoretical spacing of both TIPMI and SILAC (Figure 3 ). The experimental range has not been measured, to our knowledge, for intact protein SILAC and could not be compared. At $\sim 15 \mathrm{kDa}$ the experimentally observed range was $0.15 \%$ mass distribution (range $42-65 \mathrm{Da}$ ) for TIPMI, 3-times higher than predicted, but still 3-times lower than the lowest possible (theoretical limit of) variability of SILAC $0.47 \%$ mass distribution (range of $35-105 \mathrm{Da}$ ). All metabolic labeling techniques can result in overlap between labeled and unlabeled peaks (e.g. PTMs). Whereas SILAC-labeled intact proteins can overlap with oxidative modifications, salt-adducts, phosphorylation, and glycosylation $^{34}$ (among other PTMs); TIPMI-labeled proteins tend to overlap with oxidative 
modifications and salt-adducts with the labeling regime used in this study, but can be tuned to avoid this.

TIPMI and Native SILAC, the only nutrient-rich media techniques, result in broader isotope distributions

Broadening in TIPMI involves transitioning from the statistical regime of rare events (1.1\%

${ }^{13} \mathrm{C}$ total incorporation) and narrow distribution functions (low $\mathrm{k}$ Poisson) to the broader distribution functions expected for $9.2 \%{ }^{13} \mathrm{C}$ total incorporation (a high $\mathrm{k}$ Poisson is also a binomial distribution). Other than TIPMI, the only other method compatible with nutrientrich media was termed "Native SILAC." In this technique, a labeled amino acid is added to the media in sufficient quantity to compete with natural abundance components in the media. ${ }^{34,35}$ Native SILAC, like all SILAC approaches, is challenged by competition from labeled and unlabeled amino acids, and by arginine to proline conversion. Top-down SILAC is exponentially more susceptible to these problems than bottom-up SILAC because the amount of unlabeled protein increases in proportion to the power of the number of (replaced) amino acids. For example, an organism that is incorporating $~ 90 \%$ labeled lysine into proteins would result in a slight, $10 \%$ decrease in heavy-labeled peak intensities for trypsin proteolyzed peptides, which contain only one label, but a $65 \%$ decrease of the fully-labeled peak intensity for an intact protein with 10 lysines (probability labeled $0.90^{10}=0.35$, or $35 \%$, unlabeled $100-35=65 \%$ ). The resulting mixture of proteins with n-unlabeled arginine or lysine, or n-proline converted from arginine, result in intact protein SILAC peak broadening and asymmetry. TIPMI labelled peaks, on the other hand, generally follow a Poisson distribution and are more symmetrical (Figure 2). For sharpest labeled peak results, ${ }^{13} \mathrm{C}^{15} \mathrm{~N}$ depletion ${ }^{7}$ is selected when a defined media can be used and adequate resources are available; therefore as alternative, ${ }^{13} \mathrm{C}$-depleted-glucose is recommended for intact proteins and thinner peaks.

\section{Quantitative protein expression profiling by TIPMI}

A pseudo-gel of a representative LC-MS run has 100 Light:Heavy intact protein peak pairs easily distinguished "by-eye," without a priori knowledge of protein ID (Figure 4, 2D pseudogel). (Note: 200 unlabeled peaks were distinguished by conservative, automated peak picking throughout an LC-MS acquisition). To test the quantitative capabilities of TIPMI, natural abundance and labeled samples were mixed at varying ratios. Data analyses were manually performed, which imposed a practical limit on the number of proteins that could be analyzed. To avoid most data-selection biases, proteins above an abundance cutoff were chosen. As a result, the dynamic range of TIPMI was not assessed. To assure an adequate representation of protein mass range, the abundance cutoff was dropped until proteins from masses 8 to $47 \mathrm{kDa}$ were included, resulting in the analysis of 21 proteins (and a minimal $\mathrm{S} / \mathrm{N}$ of 19). Regression analysis of the 21 most abundant intact proteins yielded good correlation $\left(\mathrm{y}=1.05 \mathrm{x} 3.2, \mathrm{R}^{2}=0.999\right)$ between observed and expected relative peak areas across two orders of (relative concentration) magnitude, demonstrating TIPMI's utility for relative protein quantitation (Figure 5, Figure S8). 


\section{TIPMI precision and accuracy compare favorably with label-free methods}

To compare TIPMI to label-free quantitation, the same data set used above was analyzed using a variety of label-free data analysis methods (see methods section for details). The best label-free analysis had considerably decreased accuracy and precision (linear regression analysis with the same 21 proteins, 7 mixtures and 2 biological replicates: $y=1.48 x-2, R^{2}$ $=0.98$ ). A detailed analysis of the sources of variation showed that early and late eluting proteins were particularly problematic for our label-free analysis, presumably due to variability in LC retention. Removing one early and one late eluting outlier greatly improved label-free quantitation, but could not close the performance gap with TIPMI (Label-Free, outliers removed $y=1.09 x+0.36, R^{2}=0.999$, Figure 5). These outliers did not affect the TIPMI analysis, which was significantly less affected by variations in LC-retention. This study did not systematically address dynamic range (the range in $\mathrm{S} / \mathrm{N}$ of the proteins used here was 19-299), and as a result, our comparison of TIPMI to label-free analysis should not be considered as comprehensive. We also appreciate the benefits that improving our protein separation would offer to the TIPMI method. In this proof of concept, we have only skimmed the surface of the proteome, but fully anticipate the proteome depth that can be analyzed with label-free when coupling TIPMI labeling and data analysis workflow to advanced separation methods and automatic data analysis.

\section{Conclusion}

TIPMI presents the following strengths: i) low cost (less expensive sources of label and less label used), ii) predictable peak shifts, iii) applicability to most organisms, iv) compatibility with partial-labeling proteomics workflows, v) no requirement for the identity of the molecules that are being quantified, vi) does not require high resolving power MS, vii) permissible in sample preparation and chromatography variability, viii) improves quantitative accuracy and precision, and ix) fast labeling. Using low quantities of label minimizes toxicity, and the use of carbon and hydrogen, which are present in all biomolecules, could allow for applications beyond proteomics. In addition, given sufficient resolving power, the appropriate neutron encoded labels, e.g. partially deuterated water or sugar, could increase the multiplexing capacity of TIPMI. ${ }^{28,36} \mathrm{~A}$ bottleneck in our current workflow is the requirement for manual data analysis, which currently limits TIPMI's applicability to large-scale quantitation. TIPMI could however, complement large-scale label free analysis. For example, it could be used to more accurately measure the magnitude, and confirm statistically significant expression differences identified in label-free workflows. Here we have laid the groundwork for a readily accessible labeling and analysis method for top-down proteomics, and anticipate its expansion into quantitation of other biomolecules.

\section{Supplementary Material}

Refer to Web version on PubMed Central for supplementary material.

\section{Acknowledgments}

Research reported in this publication was supported by the National Institute of Neurological Disorders and Stroke of the National Institutes of Health under award number R01NS065263. We thank Dr. Barry Karger for thoughtful advice and edits, Dr. Gary V. Lopez for guidance and support in LabVIEW programing, Jennifer Poirer for initial 
experimental data, and our group members Catherine Rawlins and Daniel Donnelly for the generous feedback in this project.

\section{References}

1. Pan S, Aebersold R, Chen R, Rush J, Goodlett DR, McIntosh MW, Zhang J, Brentnall TA. Mass Spectrometry Based Targeted Protein Quantification: Methods and Applications. Journal of Proteome Research. 2009; 8(2):787-797. [PubMed: 19105742]

2. Ong SE, Blagoev B, Kratchmarova I, Kristensen DB, Steen H, Pandey A, Mann M. Stable Isotope Labeling by Amino Acids in Cell Culture, SILAC, as a Simple and Accurate Approach to Expression Proteomics. Molecular \& Cellular Proteomics. 2002; 1(5):376-386. [PubMed: 12118079]

3. McClatchy DB, Yates JR. Stable Isotope Labeling of Mammals (SILAM). Cold Spring Harbor Protocols. 2008; 2008(2) pdb.prot4940.

4. Krüger M, Moser M, Ussar S, Thievessen I, Luber CA, Forner F, Schmidt S, Zanivan S, Fässler R, Mann M. SILAC Mouse for Quantitative Proteomics Uncovers Kindlin-3 as an Essential Factor for Red Blood Cell Function. Cell. 2008; 134(2):353-364. [PubMed: 18662549]

5. McClatchy DB, Liao L, Park SK, Xu T, Lu B, Yates JR III. Differential proteomic analysis of mammalian tissues using SILAM. PLoS One. 2011; 6(1):e16039. [PubMed: 21283754]

6. Filiou MD, Varadarajulu J, Teplytska L, Reckow S, Maccarrone G, Turck CW. The $15 \mathrm{~N}$ isotope effect in Escherichia coli: a neutron can make the difference. Proteomics. 2012; 12(21):3121-8. [PubMed: 22887715]

7. Pasa-Tolic L, Jensen PK, Anderson GA, Lipton MS, Peden KK, Martinovic S, Tolic N, Bruce JE, Smith RD. High throughput proteome-wide precision measurements of protein expression using mass spectrometry. J Am Chem Soc. 1999; 121(34):7949-7950.

8. Du Y, Parks BA, Sohn S, Kwast KE, Kelleher NL. Top-Down Approaches for Measuring Expression Ratios of Intact Yeast Proteins Using Fourier Transform Mass Spectrometry. Analytical Chemistry. 2006; 78(3):686-694. [PubMed: 16448040]

9. Veenstra TD, Martinovic S, Anderson GA, Pasa-Tolic L, Smith RD. Proteome analysis using selective incorporation of isotopically labeled amino acids. Journal of the American Society for Mass Spectrometry. 2000; 11(1):78-82. [PubMed: 10631667]

10. Ong SE, Blagoev B, Kratchmarova I, Kristensen DB, Steen H, Pandey A, Mann M. Stable isotope labeling by amino acids in cell culture, SILAC, as a simple and accurate approach to expression proteomics. Mol Cell Proteomics. 2002; 1(5):376-86. [PubMed: 12118079]

11. Waanders LF, Hanke S, Mann M. Top-Down Quantitation and Characterization of SILAC-Labeled Proteins. Journal of the American Society for Mass Spectrometry. 2007; 18(11):2058-2064. [PubMed: 17920290]

12. Collier TS, Sarkar P, Rao B, Muddiman DC. Quantitative top-down proteomics of SILAC labeled human embryonic stem cells. Journal of the American Society for Mass Spectrometry. 2010; 21(6):879-889. [PubMed: 20199872]

13. Ntai I, Kim K, Fellers RT, Skinner OS, Smith ADt, Early BP, Savaryn JP, LeDuc RD, Thomas PM, Kelleher NL. Applying label-free quantitation to top down proteomics. Anal Chem. 2014; 86(10): 4961-8. [PubMed: 24807621]

14. Tran JC, Zamdborg L, Ahlf DR, Lee JE, Catherman AD, Durbin KR, Tipton JD, Vellaichamy A, Kellie JF, Li M, Wu C, Sweet SM, Early BP, Siuti N, LeDuc RD, Compton PD, Thomas PM, Kelleher NL. Mapping intact protein isoforms in discovery mode using top-down proteomics. Nature. 2011; 480(7376):254-8. [PubMed: 22037311]

15. Hellerstein MK, Neese RA. Mass isotopomer distribution analysis: a technique for measuring biosynthesis and turnover of polymers. The American journal of physiology. 1992; 263(5 Pt 1):E988-1001. [PubMed: 1443132]

16. Papageorgopoulos C, Caldwell K, Shackleton C, Schweingrubber H, Hellerstein MK. Measuring Protein Synthesis by Mass Isotopomer Distribution Analysis (MIDA). Analytical Biochemistry. 1999; 267(1):1-16. [PubMed: 9918649] 
17. Cargile BJ, Bundy JL, Grunden AM, Stephenson JL. Synthesis/Degradation Ratio Mass Spectrometry for Measuring Relative Dynamic Protein Turnover. Analytical Chemistry. 2004; 76(1):86-97. [PubMed: 14697036]

18. Previs SF, Fatica R, Chandramouli V, Alexander JC, Brunengraber H, Landau BR. Quantifying rates of protein synthesis in humans by use of $2 \mathrm{H} 2 \mathrm{O}$ : application to patients with end-stage renal disease. American Journal of Physiology - Endocrinology and Metabolism. 2004; 286(4):E665E672. [PubMed: 14693509]

19. Rachdaoui N, Austin L, Kramer E, Previs MJ, Anderson VE, Kasumov T, Previs SF. Measuring Proteome Dynamics in Vivo: AS EASY AS ADDING WATER? Molecular \& Cellular Proteomics: MCP. 2009; 8(12):2653-2663. [PubMed: 19724074]

20. Whitelegge JP, Katz JE, Pihakari KA, Hale R, Aguilera R, Gómez SM, Faull KF, Vavilin D, Vermaas W. Subtle modification of isotope ratio proteomics; an integrated strategy for expression proteomics. Phytochemistry. 2004; 65(11):1507-1515. [PubMed: 15276448]

21. Huttlin EL, Hegeman AD, Harms AC, Sussman MR. Comparison of Full Versus Partial Metabolic Labeling for Quantitative Proteomics Analysis in Arabidopsis thaliana. Molecular \& Cellular Proteomics. 2007; 6(5):860-881. [PubMed: 17293592]

22. Jehmlich N, Schmidt F, Taubert M, Seifert J, Bastida F, von Bergen M, Richnow HH, Vogt C. Protein-based stable isotope probing. Nat Protocols. 2010; 5(12):1957-1966. [PubMed: 21127489]

23. Siebers B, Schönheit P. Unusual pathways and enzymes of central carbohydrate metabolism in Archaea. Current Opinion in Microbiology. 2005; 8(6):695-705. [PubMed: 16256419]

24. Sherman F. Getting started with yeast. Methods Enzymol. 2002; 350:3-41. [PubMed: 12073320]

25. Wessel D, Flugge UI. A method for the quantitative recovery of protein in dilute solution in the presence of detergents and lipids. Anal Biochem. 1984; 138(1):141-3. http://site.ebrary.com/lib/ northeastern/Doc?id=10006728. [PubMed: 6731838]

26. Doucette AA, Vieira DB, Orton DJ, Wall MJ. Resolubilization of precipitated intact membrane proteins with cold formic acid for analysis by mass spectrometry. J Proteome Res. 2014; 13(12): 6001-12. [PubMed: 25384094]

27. Kellie JF, Catherman AD, Durbin KR, Tran JC, Tipton JD, Norris JL, Witkowski CE 2nd, Thomas PM, Kelleher NL. Robust analysis of the yeast proteome under $50 \mathrm{kDa}$ by molecular-mass-based fractionation and top-down mass spectrometry. Anal Chem. 2012; 84(1):209-15. [PubMed: 22103811]

28. Marshall AG, Senko MW, Li W, Li M, Dillon S, Guan S, Logan TM. Protein Molecular Mass to 1 Da by 13C, 15N Double-Depletion and FT-ICR Mass Spectrometry. J Am Chem Soc. 1997; 119(2):433-434.

29. Whitelegge JP, Katz JE, Pihakari KA, Hale R, Aguilera R, Gomez SM, Faull KF, Vavilin D, Vermaas W. Subtle modification of isotope ratio proteomics; an integrated strategy for expression proteomics. Phytochemistry. 2004; 65(11):1507-15. [PubMed: 15276448]

30. Huttlin EL, Hegeman AD, Harms AC, Sussman MR. Comparison of full versus partial metabolic labeling for quantitative proteomics analysis in Arabidopsis thaliana. Mol Cell Proteomics. 2007; 6(5):860-81. [PubMed: 17293592]

31. Claydon AJ, Thom MD, Hurst JL, Beynon RJ. Protein turnover: measurement of proteome dynamics by whole animal metabolic labelling with stable isotope labelled amino acids. Proteomics. 2012; 12(8):1194-206. [PubMed: 22577021]

32. Busch R, Kim YK, Neese RA, Schade-Serin V, Collins M, Awada M, Gardner JL, Beysen C, Marino ME, Misell LM, Hellerstein MK. Measurement of protein turnover rates by heavy water labeling of nonessential amino acids. Biochim Biophys Acta. 2006; 1760(5):730-44. [PubMed: 16567052]

33. Wang X, Yuan Z-F, Fan J, Karch KR, Ball LE, Denu JM, Garcia BA. A novel quantitative mass spectrometry platform for determining site-specific protein O-Glc-NAcylation dynamics. Molecular \& Cellular Proteomics. 2016

34. Collier TS, Sarkar P, Rao B, Muddiman DC. Quantitative top-down proteomics of SILAC labeled human embryonic stem cells. Journal of the American Society for Mass Spectrometry. 2010; 21(6):879-89. [PubMed: 20199872] 
35. Frohlich F, Christiano R, Walther TC. Native SILAC: metabolic labeling of proteins in prototroph microorganisms based on lysine synthesis regulation. Mol Cell Proteomics. 2013; 12(7):19952005. [PubMed: 23592334]

36. Rhoads TW, Rose CM, Bailey DJ, Riley NM, Molden RC, Nestler AJ, Merrill AE, Smith LM, Hebert AS, Westphall MS, Pagliarini DJ, Garcia BA, Coon JJ. Neutron-encoded mass signatures for quantitative top-down proteomics. Anal Chem. 2014; 86(5):2314-9. [PubMed: 24475910]

37. Sullivan, W., Ashburner, M., Hawley, RS. ebrary Inc. Drosophila protocols. Cold Spring Harbor Laboratory Press; Cold Spring Harbor, N.Y: 2000. http://site.ebrary.com/lib/northeastern/Doc? id $=10006728$

38. Collier TS, Hawkridge AM, Georgianna DR, Payne GA, Muddiman DC. Top-down identification and quantification of stable isotope labeled proteins from Aspergillus flavus using online nanoflow reversed-phase liquid chromatography coupled to a LTQ-FTICR mass spectrometer. Anal Chem. 2008; 80(13):4994-5001. [PubMed: 18512951]

39. Waanders LF, Hanke S, Mann M. Top-down quantitation and characterization of SILAC-labeled proteins. Journal of the American Society for Mass Spectrometry. 2007; 18(11):2058-64. [PubMed: 17920290]

40. Senko MW, Beu SC, McLaffertycor FW. Determination of monoisotopic masses and ion populations for large biomolecules from resolved isotopic distributions. J Am Soc Mass Spectrom. 1995; 6(4):229-33. [PubMed: 24214167] 
1:2 Mixture- unlabeled:labeled yeast

a) LC-MS BPC

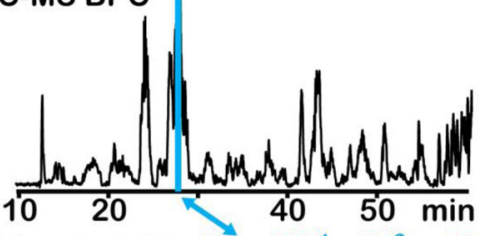

b) Extracted MS: $15 \mathrm{~s}\left(27 \frac{1}{8}-27 \frac{3}{8} \mathrm{~min}\right)$

Protease B Inhibitor 2

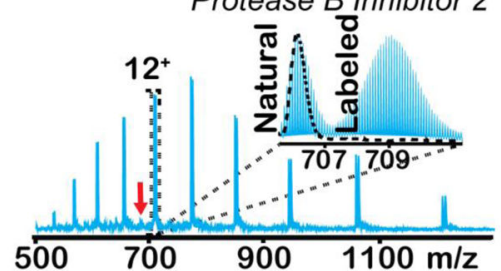

c) Deconvoluted Proteins: $10-60 \mathrm{~min}$

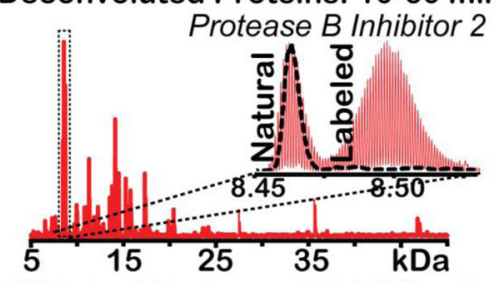

d) EIC Intact Protease B Inhibitor 2

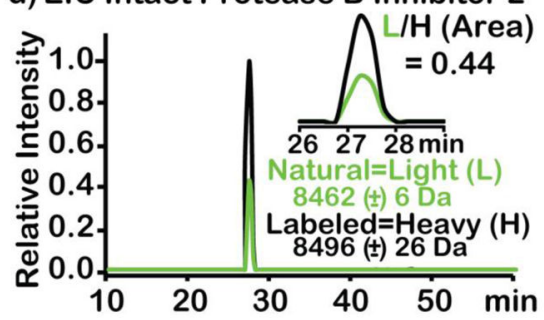

Figure 1. Adding ${ }^{13} \mathrm{C}$-glucose to nutrient-rich media increases intact protein mass and enables isotope dilution MS

Raw and deconvoluted LC-MS data and visualization of TIPMI results for the $8.4 \mathrm{kDa}$ Protease B Inhibitor 2, samples mixed 1:2 (Light:Heavy). a) Base peak chromatogram (BPC) from 10-60 minutes. b) An extracted mass spectrum (15 seconds averaged). c) Average of MaxEnt deconvoluted spectra from 10-60 minutes. d) Extracted ion chromatogram (EIC) as a function of time for intact Protease B Inhibitor 2. Similar results on a 15-fold less abundant protein (indicated with red arrow) are shown in Figure S2. 


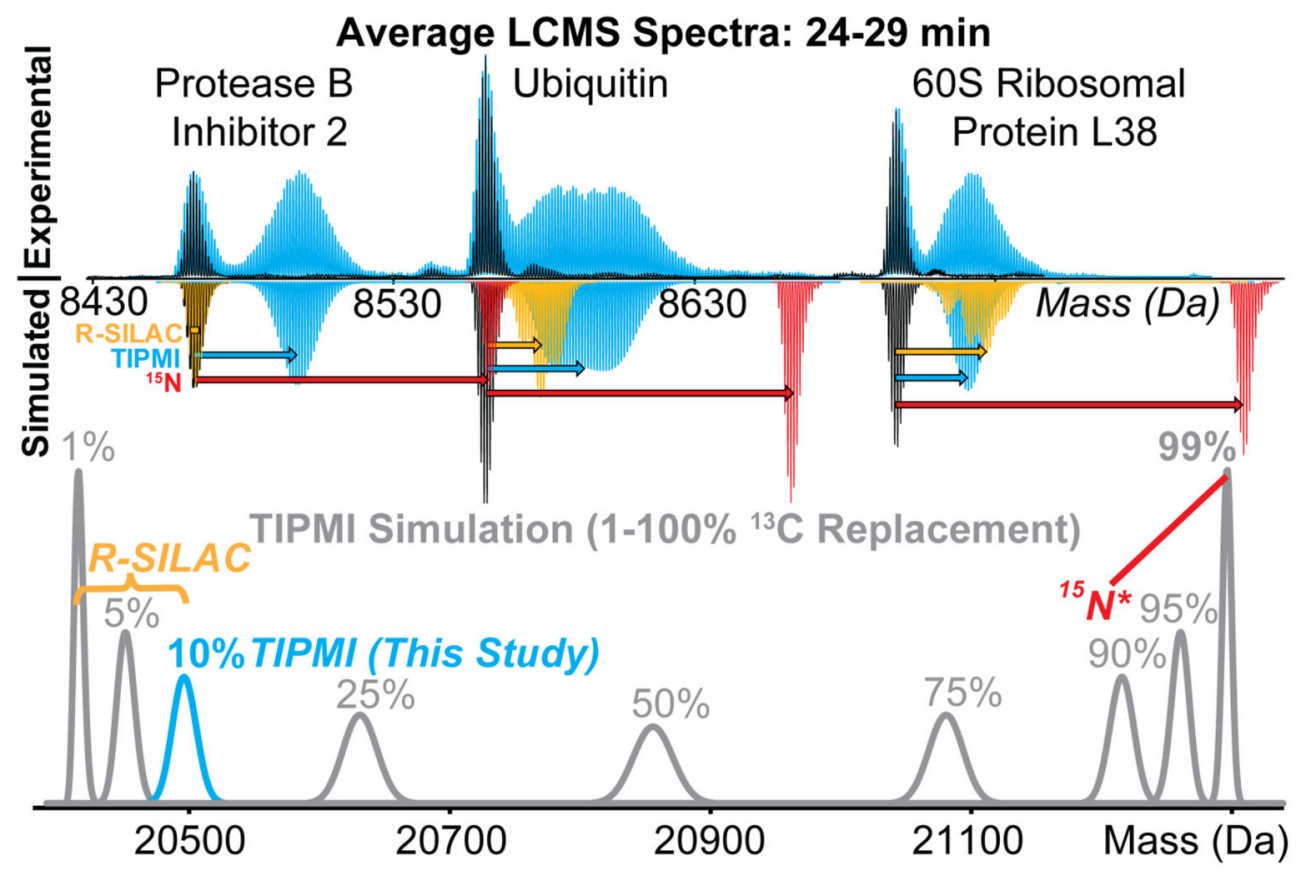

Figure 2. TIPMI creates tunable mass shifts and intact mass peak pairs that can be readily identified in protein mixtures

Top- TIPMI reliably creates resolved intact mass peak pairs, whereas SILAC does not. Experimental (facing up) LC-MS data (24-29 minutes sum) from a 1:2 (Light:Heavy) mix of yeast (blue) overlaid with natural abundance yeast (black). Inverted spectra are simulated for the two rich media techniques, TIPMI (blue spectrum) and Native ${ }^{13} \mathrm{C}_{6}$-Arg SILAC (gold spectrum), for the minimal media technique, ${ }^{15} \mathrm{~N}$ replacement (red spectrum), and simulated natural abundance (black inverted). Note that for the Protease B Inhibitor 2 protein there is no R-SILAC pair (because there is no arginine in the protein), and the ${ }^{15} \mathrm{~N}$ labeled peak overlaps with the natural abundance peak of another protein. Bottom- TIPMI can access a large range of protein mass shift, whereas SILAC or $99 \%{ }^{15} \mathrm{~N}$ total incorporation cannot. The theoretical mass shifts for each technique is calculated with respect to a $\sim 20$ $\mathrm{kDa}$ protein (specifically, the $20.4 \mathrm{kDa}$ protein, 60S Ribosomal Protein L17-A). Note that the extremes of TIPMI labeling, e.g. $\sim 0-1 \%{ }^{13} \mathrm{C}^{15} \mathrm{~N}$ depleted total and $95-99 \%{ }^{13} \mathrm{C}$ total enrichment can only be obtained in a defined media. Further details found in the Supporting Information (S-3). *Complete ${ }^{15} \mathrm{~N}$ incorporation results in different (roughly $1 / 3$ of the) mass shifts than complete ${ }^{13} \mathrm{C}$ incorporation. 


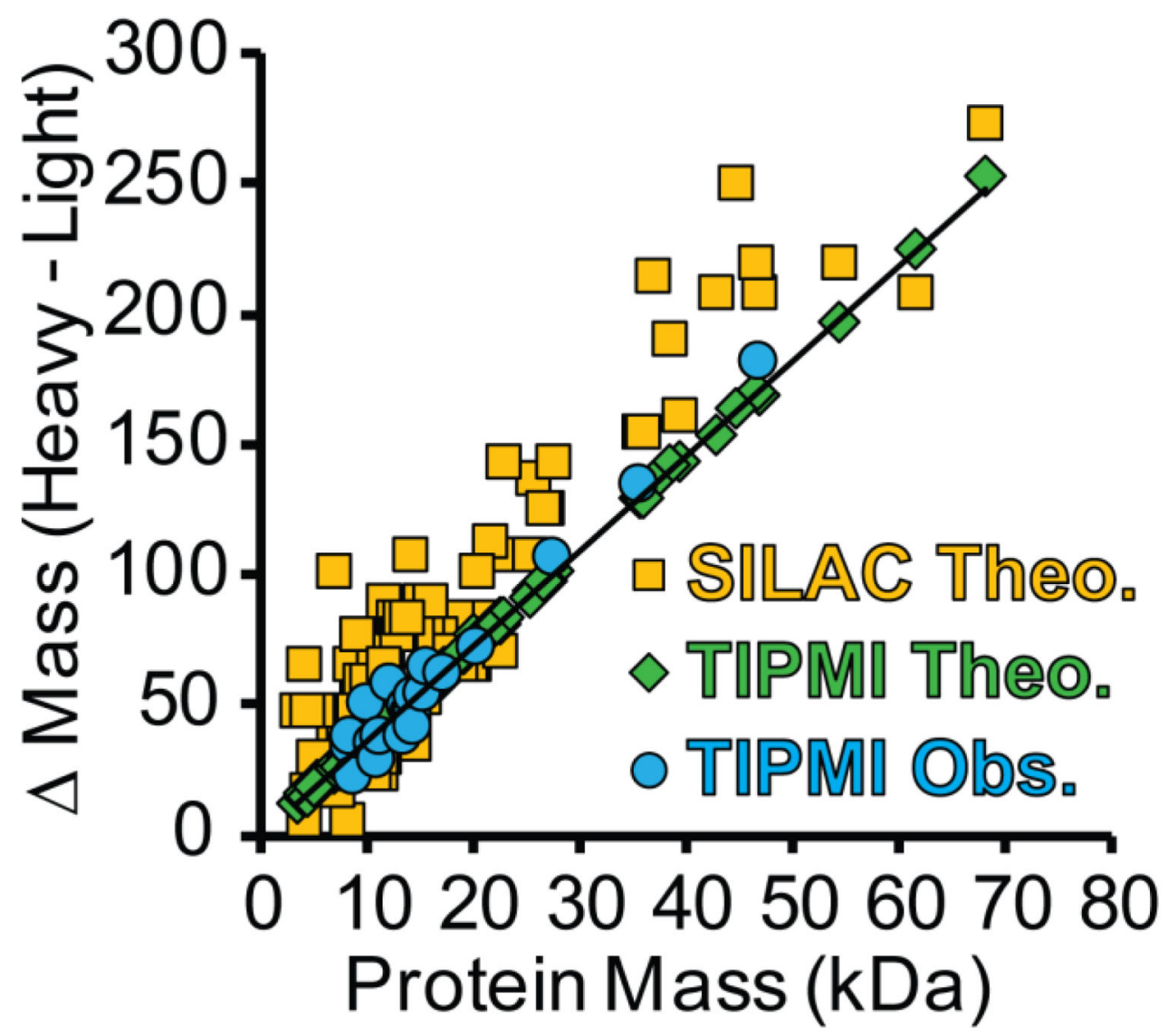

Figure 3. TIPMI peak pair separations are more predictable than those of SILAC pairs Delta mass between light and heavy peaks are plotted for SILAC $\left({ }^{13} \mathrm{C}\right.$-Lysine theoretical, gold), $8.1 \%{ }^{13} \mathrm{C}$ new incorporation TIPMI, theoretical (green), and experimental (blue). 153 proteins whose ID's were reported by Kellie et al. were used for theoretical data. The 21 proteins used for the TIPMI plot are listed in Table S1, and are the same proteins analyzed throughout this study. 


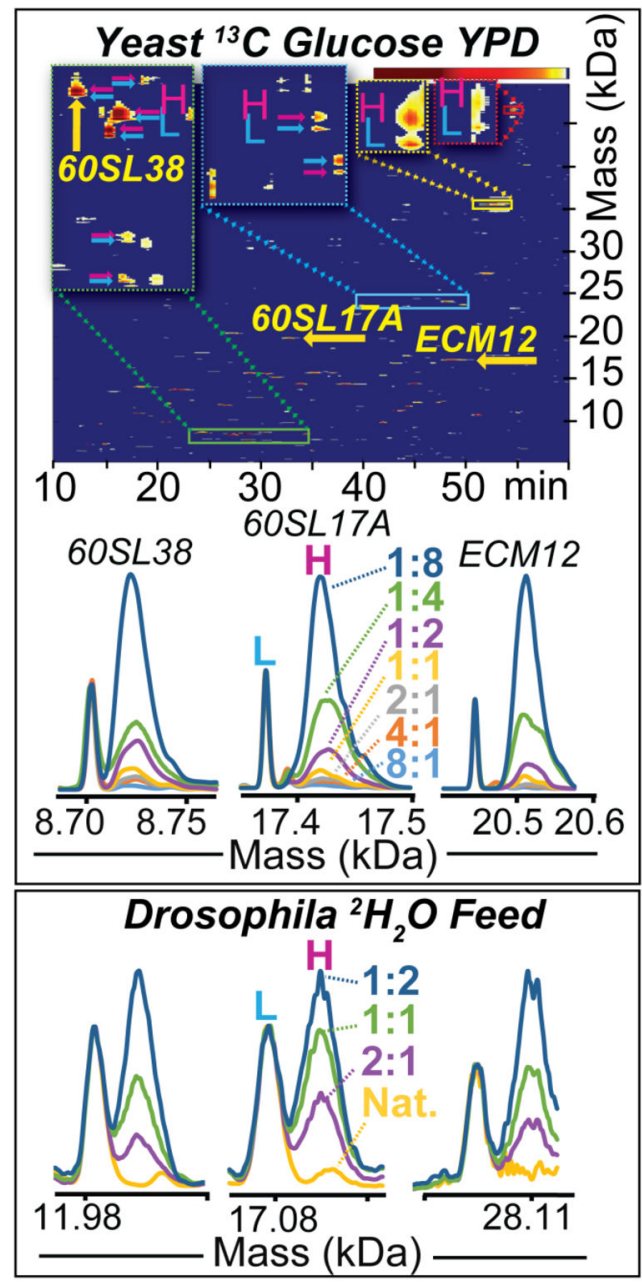

Figure 4. TIMPI ${ }^{13} \mathrm{C}$ and ${ }^{2} \mathrm{H}$ isotope dilution in a microbe and an animal model

Top- MS analysis in the form of a deconvoluted intact protein 2D pseudo-gel view. Inset panels are expansions of like-colored boxes, to better illustrate Light:Heavy peak pairs (L and $\mathrm{H}$ indicated by blue and pink arrows, respectively). Middle- Mass spectra of seven different L:H mixtures for three proteins from the yeast Saccharomyces cerevisiae labeled with ${ }^{13} \mathrm{C}$ sugar, with heavy proteins tuned $\sim 0.3 \%$ by mass. Bottom- Mass spectra of four different L:H mixtures for three (unknown) proteins of Drosophila melanogaster, labeled with ${ }^{2} \mathrm{H}_{2} \mathrm{O}$ water. Peak shifts as a function of amount of ${ }^{2} \mathrm{H}_{2} \mathrm{O}$ and ${ }^{13} \mathrm{C}$ sugar labels are shown for Escherichia coli in Figure S6. 


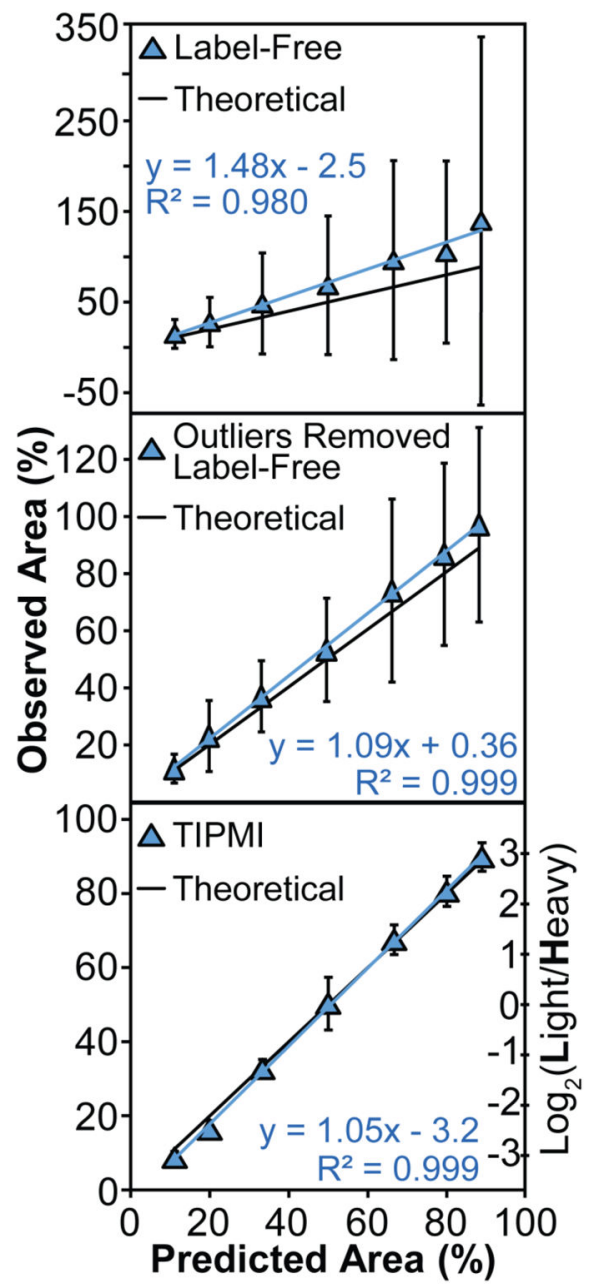

Figure 5. TIPMI-enabled quantitation of intact proteins across two orders of magnitude of relative concentration

For these experiments light- and heavy-labeled yeast were mixed in $\mathrm{L} / \mathrm{H}$ ratios of 1:8, 1:4, $1: 2,1: 1,2: 1,4: 1,8: 1$ by relative cell count, and peak areas (both light and heavy) for the 21 most abundant proteins were calculated. The peak areas (two biological replicates) were quantified by TIC-normalized absolute intensity (top, middle), or TIPMI (bottom). Labelfree methods could be improved by omitting early- and late-eluting protein outliers (middle). Details of this comparison are given in Figure S4. 